\title{
“Talleres para potenciar el pensamiento numérico, métrico y Geométrico en estudiantes de séptimo grado de educación Básica secundaria"1
}

\author{
“Workshops para melhorar o pensamento numérico, \\ métricas e geométrico em alunos da sétima \\ série do ensino secundário básico"
}

“Workshops para melhorar o pensamento numérico, métricas e geométrico em alunos da sétima série do ensino secundário básico"

Recibido: mayo de 2013

Aprobado: agosto de 2013
July Karina Celis Beltrán ${ }^{2}$

Liana Prakrity Moreno Acuña ${ }^{3}$ Carlos Bautista Duque ${ }^{4}$

\section{Resumen}

Los estudiantes presentan algunos obstáculos en el área de las matemáticas, específicamente en la solución de problemas: Comprensión de lectura; Manejo del lenguaje matemático formal, al leerlo y escribirlo; Construcción de conceptos matemáticos; La aplicación de conceptos matemáticos en la solución de problemas en diferentes contextos. Propusimos un trabajo enfocado en la solución de problemas; en donde se buscaba potenciar el pensamiento métrico, geométrico y numérico, además de fortalecer: La apropiación y dominio del Lenguaje Matemático; La habilidad para justificar o refutar conjeturas, la argumentación coherente; La ejecución segura y rápida de algoritmos. Finalizamos describiendo algunas fortalezas y debilidades identificadas durante la orientación de este proceso de enseñanza y aprendizaje.

Palabras clave: Talleres; otras nociones de Educación Matemática; resolución de problemas; matemáticas escolares; geometría; medida; números; aprendizaje; procesos cognitivos; pensamiento matemático.

\section{Abstract}

Students present some obstacles in the area of mathematics, specifically in problem solving: Reading Comprehension; Management of formal mathematical language, to read and write; Construction of mathematical concepts; The application of mathematical concepts in solving problems

$1 \quad$ Articulo de Investigación

2 Universidad Industrial de Santander. Colombia. Contacto: karinitacelis@yahoo.es

3 Universidad Industrial de Santander. Colombia. Contacto: lianaprakrity@hotmail.com

4 Universidad Industrial de Santander. Colombia. Perteneciente al Grupo Educación Matemática, EDUMAT -UIS.

Contacto: carlosbautistaduque@gmail.com 
in different contexts. Proposed a work focused on solving problems, in which we wanted to enhance the thinking metric, geometric and numerical, and strengthen: Domain Ownership and Mathematical Language; The ability to justify or refute conjectures, coherent argument; The safe and fast implementation algorithms. We conclude by describing some strengths and weaknesses identified during the orientation of this teaching and learning process.

Keywords: Workshops, other notions of mathematics education, problem solving, school mathematics, geometry, measurement, numbers, learning cognitive processes mathematical thinking.

\section{Resumo}

Os alunos apresentam alguns obstáculos na área de matemática, especificamente na solução de problemas: compreensão da leitura; Gestão da linguagem matemática formal, a ler e escrever; Construção de conceitos matemáticos. A aplicação de conceitos matemáticos na resolução de problemas em diferentes contextos. Propôs um trabalho focado na resolução de problemas, em que queríamos aumentar o pensamento métrica, geométrica e numérica, e fortalecer: a propriedade do domínio e linguagem matemática. A capacidade de justificar ou refutar conjecturas, argumentação coerente. Os algoritmos de implementação segura e rápida. Concluímos descrevendo alguns pontos fortes e fracos identificados durante a orientação do processo de ensino e aprendizagem.

Palavras-chave: Workshops, outras noções de educação matemática, resolução de problemas, a matemática da escola, geometria, medição, números, aprendendo processos cognitivos pensamento matemático.

\section{Introducción}

En nuestra experiencia en el Servicio Social Educativo I, con estudiantes de los colegios Escuela Normal Superior de Bucaramanga, Institución Educativa las Américas y el Programa de Asesoría para el Mejoramiento del Rendimiento Académico (PAMRA) de la universidad Industrial de Santander, tuvimos la oportunidad de observar y analizar algunos de los obstáculos que se presentan en los jóvenes durante su proceso de aprendizaje; particularmente en el tema de solución de problemas.

Algunos obstáculos que se contemplan son:

- Distraerse, con el uso inadecuado de la tecnología (celulares, BlackBerry, iPod, iPhone),
- Exceso de confianza, los estudiantes creen saber cosas que no saben y no se permiten cuestionar o examinar.

- Prejuicio cognitivo, no se permiten preguntar para aclarar sus dudas por miedo a ser juzgados ya sea por el profesor o por los compañeros de clase.

- Bajo Interés por resolver las situaciones problemas planteadas.

- Comprensión de lectura, se presenta dificultad para manejar la simbología o lenguaje matemático (Leer y escribir)

- Dificultad al construir y apropiarse de conceptos matemáticos.

- Dificultad para planear y ejecutar estrategias de solución. 
Teniendo en cuenta los aspectos anteriores, diseñamos e implementamos ciertos talleres que no solo despierte el interés de los estudiantes en la asignatura, sino que también potencialicen tres pensamientos matemáticos (Pensamiento Métrico, Numérico y Geométrico) por medio de la solución de problemas, además que el estudiante pueda aprender de manera significativa la utilidad de las matemáticas.

\section{Marco de referencia conceptual}

En referencia a la resolución de problemas: "Las situaciones problemas proporcionan el contexto inmediato en donde el quehacer matemático cobra sentido, en la medida en que las situaciones que se aborden estén ligadas a experiencias cotidianas y, por ende, sean más significativas para los alumnos. Estos problemas pueden surgir del mundo cotidiano cercano o lejano, pero también de otras ciencias y de las mismas matemáticas, convirtiéndose en ricas redes de interconexión e interdisciplinariedad" (Estándares Básicos en Competencias Matemáticas, 2006, p.52).

El pensamiento numérico y los sistemas numéricos.

"Los Lineamientos Curriculares de Matemáticas plantean el desarrollo de los procesos curriculares y la organización de actividades centradas en la comprensión del uso y de los significados de los números y de la numeración; la comprensión del sentido y significado de las operaciones y de las relaciones entre números, y el desarrollo de diferentes técnicas de cálculo y estimación. Dichos planteamientos se enriquecen si, además, se propone trabajar con las magnitudes, las cantidades y sus medidas como base para dar significado y comprender mejor los procesos generales relativos al pensamiento numérico y para ligarlo con el pensamiento métrico" (Estándares Básicos en Competencias Matemáticas,2006, p.58).
El pensamiento espacial y los sistemas geométricos.

"En los sistemas geométricos se hace énfasis en el desarrollo del pensamiento espacial, el cual es considerado como el conjunto de los procesos cognitivos mediante los cuales se construyen y se manipulan las representaciones mentales de los objetos del espacio, las relaciones entre ellos, sus transformaciones, y sus diversas traducciones a representaciones materiales. Los sistemas geométricos se construyen a través de la exploración activa y modelación del espacio tanto para la situación de los objetos en reposo como para el movimiento. Esta construcción se entiende como un proceso cognitivo de interacciones, que avanza desde un espacio intuitivo o sensorio-motor (que se relaciona con la capacidad práctica de actuar en el espacio, manipulando objetos, localizando situaciones en el entorno y efectuando desplazamientos, medidas, cálculos espaciales, etc.), a un espacio conceptual o abstracto relacionado con la capacidad de representar internamente el espacio, reflexionando y razonando sobre propiedades geométricas abstractas, tomando sistemas de referencia y prediciendo los resultados de manipulaciones mentales" (Estándares Básicos en Competencias Matemáticas,2006, p.61).

El pensamiento métrico y los sistemas métricos o de medidas.

"Los conceptos y procedimientos propios de este pensamiento hacen referencia a la comprensión general que tiene una persona sobre las magnitudes y las cantidades, su medición y el uso flexible de los sistemas métricos o de medidas en diferentes situaciones. Especificando conceptos y procedimientos relacionados con este tipo de pensamiento, como:

- La construcción de los conceptos de cada magnitud.

- La comprensión de los procesos de conservación de magnitudes. 
- La estimación de magnitudes y los aspectos del proceso de "capturar lo continuo con lo discreto".

- La apreciación del rango de las magnitudes.

- La selección de unidades de medida, de patrones y de instrumentos.

- La diferencia entre la unidad y el patrón de medición.

- La asignación numérica.

- El papel del trasfondo social de la medición" (Estándares Básicos en Competencias Matemáticas, 2006, p.63).

Los cinco procesos generales de la actividad matemática

- Formular y resolver problemas

- Modelar procesos y fenómenos de la realidad

- Comunicar; razonar y formular

- Comparar y ejercitar procedimientos y algoritmos. (Estándares Básicos en Competencias Matemáticas, 2006, p.63).

\section{Metodología}

El trabajo que se realizó fue una experiencia de aula con 15 estudiantes del Instituto Educativo las Américas de Bucaramanga, del grado séptimo, se partió de una prueba diagnóstica escrita, con problemas enmarcados en los cinco Pensamientos Matemáticos. Basados en lo anterior se aplicaron cuatro talleres donde los problemas estaban enmarcados en los Pensamientos: Métrico, Geométrico y Variacional, usando como herramienta las Pruebas Saber, con el fin de saber cómo el estudiante realiza el proceso de formular y resolver problemas; modela procesos y fenómenos de la realidad; comunicar; razona, compara y ejercita procedimientos y algoritmos.

\section{Análisis de datos}

\section{Pensamiento métrico.}

Los estudiantes tienen dominio de las relaciones entre distancias y unidades de capacidad tal como galón $/ \mathrm{km}$, pero dificultad en la transformación de medidas de longitud, significado de términos matemáticos tal como arista, vista frontal en una construcción geométrica, la definición de área para un rectángulo, transforman unidades de masa, encuentran el área y el volumen de un cubo, presentan dificultad en el manejo de los conceptos de perímetro, volumen y área de una construcción formada por varios cubos, no encuentran las dimensiones del lado de un cubo cuando se conoce su volumen, los estudiantes saben que los lados de un triángulo equilátero tienen igual medida y a partir del perímetro encuentran el valor del lado, de otro modo se dificulta la aplicación del concepto de área además del uso del Teorema de Pitágoras para encontrar la altura que es desconocida en triángulos rectángulos, algunos encuentran el área sombreada en una figura, usando el área del cuadrado y del círculo, es prudente aclarar que la mayoría no logró hallar el área del círculo debido a que se le dificultan las operaciones aritméticas con números decimales, encuentran las dimensiones del rectángulo cuando se conoce el perímetro y la relación entre sus lados (la base mide el triple de la altura), persiste la dificultad al encontrar el área superficial y el volumen del paralelepípedo y de un cilindro con tapa.

\section{Pensamiento numérico.}

Los estudiantes interpretan fracciones propias de manera aritmética y geométrica, sin embargo no utilizan los resultados de las operaciones para responder la pregunta del problema, Se dificulta la solución de problemas que requiera encontrar la relación entre los datos (que la edad del padre sea el triple de la de su hijo), los estudiantes logran dar respuesta a los problemas usando el método de ensayo y error sin mostrar evidencia del uso de un lenguaje formal matemático, además se logra evidenciar dificultad en la operación con números 
racionales. Se nota destreza en la solución de problemas con números naturales; en cuanto al uso de operaciones con números racionales su desempeño no fue satisfactorio, se infiere que se debe a la falta de compresión de la situación problema y análisis de la información dada.

\section{Pensamiento geométrico.}

Existe un avance significativo en el tema de simetría. Encuentran las coordenadas de algunos puntos simétricos usando el plano cartesiano, pero en algunos casos no diferencian entre las abscisas y ordenadas también se notó poca destreza en la traslación objeto usando el plano cartesiano

\section{Conclusiones}

Potenciar los Pensamientos Matemáticos es un proceso, que debe realizarse de manera continua desde los primeros años de escolaridad, basados en la aplicación de problemas que contribuyan significativamente en darles sentido y utilidad a las Matemáticas, además de reafirmar y precisar conceptos teóricos adquiridos en clase. Sin dejar atrás la importancia que tiene establecer una relación de respeto que permita al estudiante expresar sus opiniones, razonamientos o dudas y de esta forma enriquecer el proceso de enseñanza aprendizaje.

\section{Referencias}

Jaimes. M. \& Córdoba. Y. (2.008). El juego en el desarrollo del pensamiento numérico en estudiantes de séptimo grado. Trabajo de grado Especialización en Educación Matemática, Escuela de Matemáticas, Universidad Industrial de Santander, Bucaramanga.

MEN, Ministerio de Educación Nacional. (2.006). Estándares Básicos de Competencias en Matemáticas. Santafé de Bogotá, Recuperado de: http://www.mineducacion.gov.co/ cvn/1665/articles-116042_archivo_pdf2.pdf

MEN, Ministerio de Educación Nacional. (1.998). Lineamientos Curriculares. Santafé de Bogotá, Recuperado de: http://www.mineducacion.gov.co/cvn/1665/articles-89869_ archivo_pdf9.pdf

Icfes. (2009). Pruebas Saber Pro, Matemáticas 1, 5, Ministerio de Educación Nacional, Santafé de Bogotá.

Icfes. (2009). Pruebas Saber Pro, Matemáticas 1, 5, Ministerio de Educación Nacional, Santafé de Bogotá. 